\title{
High-risk Human Papillomavirus Detection in Oropharyngeal Cancers: Comparison of Saliva Sampling Methods
}

Kai Dun Tang, PhD ${ }^{1}$, Liz Kenny, MBBS FRANZCR FRACR ${ }^{2}$, Ian H. Frazer, MB ChB(Edin) MD(Melb) FRCP(Ed) FRCPA ${ }^{3}$ and Chamindie Punyadeera, $\mathrm{PhD}^{1^{*}}$

1. The School of Biomedical Sciences, Institute of Health and Biomedical Innovation, Queensland University of Technology, Kelvin Grove, QLD, Australia and the Translational Research Institute, Brisbane, Australia.

2. School of Medicine, University of Queensland; Royal Brisbane and Women's Hospital, Brisbane; Central Integrated Regional Cancer Service, Queensland Health, Queensland, Australia.

3. Faculty of Medicine, The University of Queensland, Translational Research Institute, Brisbane, Australia.

*Corresponding author:

Associate Professor Chamindie Punyadeera

Address: The School of Biomedical Sciences, Room 603D, Institute of Health and Biomedical Innovations, Queensland University of Technology, 60 Musk Avenue, Kelvin Grove, QLD 4059, Australia.

Telephone: +61 $731380830 \quad$ Fax: +61 731386030

Email: chamindie.punyadeera@qut.edu.au

This is the author manuscript accepted for publication and has undergone full peer review but has not been through the copyediting, typesetting, pagination and proofreading process, which may lead to differences between this version and the Version of Record. Please cite this article as doi: $10.1002 /$ hed.25578

This article is protected by copyright. All rights reserved. 


\begin{abstract}
Background: Accumulating evidence has suggested the utility of salivary oral rinse as a diagnostic fluid to detect oral human papillomavirus (HPV) DNA, but there are many methods for collecting saliva.
\end{abstract}

Methods: Salivary oral rinse and unstimulated whole mouth saliva (UWMS) samples were collected from 45 oropharyngeal cancer (OPC) patients.

Results: We show a positive correlation of HPV-16 E2 $(r=0.95, \mathrm{p}<0.0001)$ and E6/7 $(r=$ 0.93, $\mathrm{p}<0.0001$ ) relative copy number as well as HPV genotypes in both sample methods. There was a significant correlation between the two sample methods in the ratio of HPV16 E2 to E6/7 DNA ( $r=0.46, p<0.01)$. Consistent with previous studies, a mixed HPV-16 form (episomal and integrated) was commonly found in both saliva and tumour samples.

Conclusion: Detection of HPV in saliva samples collected by either method yielded comparable results, and showed good sensitivity for detection of HPV derived from OPC.

Keywords: Human papillomavirus, physical status, salivary oral rinse, unstimulated whole mouth saliva, oropharyngeal cancer and non-invasive biomarker.

This article is protected by copyright. All rights reserved. 


\section{Introduction}

Oropharyngeal cancer (OPC) in which malignant cells form at the tonsillar area, the base of tongue and the oropharynx is one of the most common subsets of head and neck squamous cell carcinomas (HNSCC), accounting for an estimated 97,000 deaths annually globally ${ }^{1}$. Traditionally, HNSCC is often associated with excessive tobacco and alcohol consumption ${ }^{2}$. However, over the past two decades, there has been a dramatic rise in the incidence of OPC associated with high-risk human papillomavirus (HPV) infection in developed countries ${ }^{3}$. This may reflect an increasing number of sexual partners, the practice of oral sex, and poor oral health ${ }^{4}$.

Nearly 205 divergent HPV genotypes have been identified, of which HPV type 16 (HPV-16) is associated with approximately $90 \%$ of HPV-positive OPC cases ${ }^{5}$. In addition to HPV-16, other high risk HPV genotypes 18, 31, 33, 45, 52 and 58 are also associated with a minority of OPC cases ${ }^{6}$. Recent reports document that the majority of HPV-positive OPC patients (66\%) tend to present with more advanced disease including a high frequency of nodal metastases ${ }^{7,8}$. Nevertheless, these patients have a relatively better 5 -year survival rate, which is reported at $82 \%$, whilst it is $35 \%$ in HPV-negative HNSCC ${ }^{9}$.

This article is protected by copyright. All rights reserved. 
Emerging evidence supports that persistent infection with high-risk HPV is strongly associated with the development and progression of cervical cancer, anal cancer and OPCs ${ }^{10}$. In OPC, in contrast to cervical carcinoma, an elevated HPV-16 viral load is not significantly correlated to advanced stage disease ${ }^{11}$. In addition, the integration of the HPV DNA into the host genome is commonly associated with the development of malignant lesions, including $\mathrm{OPC}^{12}$. Integration often results in the disruption of the E2 open reading frame (ORF), which in turn leads to the upregulation of HPV E6 and E7 expression ${ }^{13}$. However, the exact mechanism of HPV DNA integration in both cervical carcinoma and OPC remains to be elucidated.

14,15 Previous studies have reported that the presence of HPV DNA in salivary oral rinse samples is positively correlated with HPV DNA in the tumour tissues and serum collected from OPC patients. Furthermore, the use of salivary HPV as a biomarker to monitor disease response and tumour recurrence in OPC patients has also been suggested ${ }^{14}$. Most of the previously published work indicates that salivary oral rinse is the best sampling method to determine the HPV status in OPC patients, when compared to oral cytobrush or swab ${ }^{16,17}$. However, little attention has been paid to in the use of unstimulated whole mouth saliva (UWMS) for this purpose. The first aim of this study was to assess the HPV DNA prevalence and type distribution in salivary oral rinse and UWMS samples. Secondly, we evaluated the HPV-16 physical status in saliva collected from OPC patients. Our findings indicate that the detection of HPV in saliva samples collected by either method yielded comparable results, and demonstrated good sensitivity for detection of HPV derived from OPC. 


\section{Materials and Methods}

\section{Study design}

This study was approved by the University of Queensland Medical Ethical Institutional Board [HREC No: 2014000679 and 2014000862]; Queensland University of Technology [HREC No: 1400000617 and 1400000641] and by the Princess Alexandra Hospital Ethics Review Board [HREC Number: HREC/12/QPAH/381]. Forty-five patients who had been diagnosed with OPC tumours were recruited from the Princess Alexandra Hospital, Woolloongabba, Queensland, Australia. Clinical stages of HNSCC patients were classified according to the American Joint Committee on Cancer (AJCC, version 7) based on Tumour-Nodal-Metastasis (TNM). All participants gave written informed consent, prior to sampling.

This article is protected by copyright. All rights reserved. 


\section{Salivary oral rinse and unstimulated whole mouth saliva sample collection and processing}

Salivary oral rinse and UWMS samples were collected from participants as described in our previous studies ${ }^{18}$. For UWMS samples, participants were asked to pool saliva in the mouth (2-5 minutes) before drooling into Falcon tube. For salivary oral rinse samples, participants were asked to swish and gargle for 1-2 minutes with 2 X $10 \mathrm{ml} 0.9 \%$ saline. All the samples were immediately frozen and kept on dry ice and delivered to the laboratory for downstream processing.

\section{DNA isolation}

Total DNA was extracted from UWMS and oral rinse samples using QIAmp DNA Mini Kit (Qiagen, Germantown, MD, USA) as per manufacturer's protocol. Briefly, $200 \mu \mathrm{L}$ of lysis buffer was added to the samples with Proteinase $\mathrm{K}$ and incubated at $56^{\circ} \mathrm{C}$ for 10 minutes. Then, $200 \mu \mathrm{L}$ of $100 \%$ Ethanol was added to the mixture and subsequently transferred to QIAmp DNA mini spin columns and following the manufacturer's instructions.

\section{qPCR analysis}

All the DNA samples $(\mathrm{n}=45)$ were diluted to $20 \mathrm{ng} / \mu \mathrm{l}$ and $50 \mathrm{ng}$ was used in duplicate in qPCR. qPCR was carried out with the QuantStudio ${ }^{\text {TM }} 7$ Flex Real-Time PCR System (Applied Biosystems, Foster City, CA, USA). Sense and anti-sense primers targeted against the HPV-16 E2 and E6/7 regions are listed in Supplementary Table S1. Human $\beta$-globin was used as an internal control. HPV-16 E2 and E6/E7 DNA standard calibration curves were 
generated by using qPCR, respectively by plotting threshold cycle (Ct values) against the logarithm of the copy number of sevenfold serially diluted Caski-derived DNA. Each of the 7 point dilutions had the same amount of total DNA by spiking with HPV-16 negative HNSCC cell line DNA (SCC-9). For HPV type distribution, ten out of thirty HPV-16 positive OPC patients were selected. Hybribio 14 high risk-HPV real time PCR was used to amplify HPV16, HPV-18 and 12 other high risk HPV genotypes (HPV 31, 33, 35, 39, 45, 51, 52, 56, 58, 59, 66 and 68), as well as $\beta$-globin, as described previously ${ }^{19}$. Note that, samples were classified as HPV positive when there was a Ct of either E2 or E6/7, and HPV negative when both E2 and E6/7 Ct values were undetermined: these were assigned a value of 40.

\section{Physical status of HPV-16}

HPV-16 physical status in both saliva fractions and tumour samples collected from HPV-16 positive OPC patients $(n=30)$ was classified based on the fold change difference between the relative copy numbers of E2 and E6/7 as described previously ${ }^{20}$. HPV-16 was classified as episomal when the Ct of both E2 and E6/7 was similar (fold change < 2.0); mixed status with an increased E2 Ct (fold change > 2) and pure integrated status when the E2 Ct was absent.

\section{Statistical analysis}

This article is protected by copyright. All rights reserved. 
The Cohen's kappa coefficient with 95\% confidence interval was used to measure the interrater agreement between salivary HPV-16 DNA testing and p16INK4a expression. https://www.graphpad.com/quickcalcs/kappa1.cfm. Sensitivity, specificity, positive predictive values (PPV), negative predictive values (NPV), Youden’s index and their $95 \%$ confidence intervals were calculated. The Spearman correlation coefficient was used to investigate the relationship of the HPV-16 DNA prevalence as well as fold change difference between E2 and E6/7 relative copy number in both saliva fractions. P-values less than 0.05 were considered significant. All the statistical analysis were performed using GraphPad Prism 7 software version 7 (GraphPad Software Inc., USA).

This article is protected by copyright. All rights reserved. 


\section{Results}

\section{Population characteristics}

OPC patients $(n=45)$ were recruited in this study (Table 1). Most of the participants were male $(93.3 \%)$ and the mean age of this population was 60.4 years ( $\mathrm{SD}=10.6,20-92)$. The majority of the patients were Caucasian (95.6\%) and current or ex-smokers (68.9\%). According to the TNM classification, OPC patients staging was as follows: Stage III (20.0\%) and IV (80.0\%). OPC tumours were predominately found in tonsil (57.8\%) and most were considered to be moderately or poorly differentiated squamous cell carcinoma (57.8\%). Tumour biopsies from 34 OPC patients were p16INK4a-positive on immunohistochemistry (IHC) analysis, 10 were negative, and one was not tested.

\section{HPV DNA prevalence and type distribution in salivary oral rinse and unstimulated} whole mouth saliva samples

Previous studies have demonstrated that overexpression of p16INK4a in the tumour tissues is strongly correlated with the detection of HPV-16 in salivary oral rinse samples collected from OPC patients ${ }^{15,18}$. However, HPV DNA prevalence and type distribution in UWMS remain unknown. To address this question, we first focused on the presence of HPV-16 E2 and E6/7 DNA in salivary oral rinse and UWMS samples. HPV-16 DNA was detected in both types of saliva sample collected from the majority of p16INK4a-positive OPC patients ( $\mathrm{n}=29,85 \%)$, from one patient with unknown p16INK4a status, and from none of the p16INK4a-negative OPC patients $(\mathrm{n}=10)$. HPV DNA detection had a sensitivity of $85 \%$ and specificity of 
$100 \%$ for prediction of tumour p16INK4a expression (kappa $=0.73 ; 95 \% \mathrm{CI}, 0.51$ to 0.94 ) as shown in Supplementary Table S3.

Between the two methods of saliva collection from OPC patients, there was a good correlation for the relative amounts of HPV16 E2 $(r=0.95, p<0.0001)$ and also of HPV16 E6/E7 ( $r=0.93, p<0.0001$ ) (Figure $1 \mathrm{~A}$ and B). Similarly, the ratio of relative amounts of HPV16 E2 to HPV16 E6/E7 correlated significantly between saliva samples collected by the two methods ( $\mathrm{r}=0.46, \mathrm{p}=0.0098$ ). Two available tumour samples were concordant with their respective saliva samples.

HPV type distribution was also concordant between the two sample methods, for 10 tested samples (Table 2).

\section{HPV-16 physical status in saliva collected from patients with oropharyngeal cancer}

HPV-16 DNA integration into somatic DNA is commonly seen in OPC and is associated with amplification of the E6/E7 region and interruption or loss of the E2 ORF ${ }^{12}$. Therefore, we measured the ratio of E6/7 DNA copy number to HPV16 E2 DNA copy number in saliva samples and classified those where the ratio was $>2$ as indicative of integrated DNA, and those where there was no E2 as indicative of loss of episomal DNA. The majority of samples collected by either method showed evidence of integrated DNA with a minority of samples consistent with only episomal DNA or only integrated DNA (Table 3). Two tested tumour samples had ratios consistent with the presence of integrated DNA.

This article is protected by copyright. All rights reserved. 


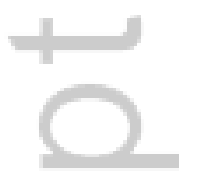

This article is protected by copyright. All rights reserved. 


\section{Discussion}

Unlike other cancer types, OPC biopsy collection is challenging as the tumours are usually small and inconspicuous. Therefore, a non-invasive sampling method is imperative to overcome this unmet clinical need. In this study, the HPV DNA prevalence, type distribution and physical status in both saliva fractions have shown a positive correlation using two different qPCR approaches. In addition, the HPV-16 physical status in both saliva and tumour tissue samples were correlated. More importantly, our results indicate that the salivary HPV16 E2/E6/7 DNA can be used as a non-invasive biomarker to determine HPV-16 physical status in OPC patients.

Human saliva is a unique body fluid which has been widely used as a non-invasive diagnostic medium for detecting both oral and systemic events by virtue of convenience sampling and being relatively inexpensive ${ }^{21,22}$. Accumulating evidence supports the notion that salivary testing can be used to predict the high risk HPV status in OPC patients ${ }^{15}$. Ang et al. reported that the p16INK4a expression, a HPV surrogate marker is positively correlated with the HPV infection in OPC tumours ${ }^{23}$. Recent work from our lab has shown that the presence of HPV16 DNA in salivary oral rinse samples is strongly correlated with p16INK4a-positivity in HNSCC patients ${ }^{18}$. This is in concordance with our current study comparing the p16INK4a expression and HPV-16 status in OPC patients.

However, there is a limitation of using saliva as the amount and quality of extracted DNA may be insufficient for the HPV testing. Among all the salivary sampling methods, UWMS showed the highest DNA yield as reported by previous studies ${ }^{16}$. To our knowledge, there is lack of study comparing HPV DNA prevalence, type distribution and physical status present 
in both saliva fractions. Only one study has reported that there is no significant difference in term of the number of HPV sequences in both saliva fractions ${ }^{24}$. In agreement with the study of Wang et al, we observed a positive correlation between both saliva fractions for HPV-16 DNA viral load and type distribution.

Accumulating studies have demonstrated that the physical status (episomal or integrated) of HPV-16 DNA can be assessed by qPCR targeting E2 and E6 ${ }^{25-27}$. Notably, a mixed HPV-16 status with the co-existence of both episomal and integrated forms is commonly detected in the tumour biopsies of OPC patients ${ }^{28}$. Consistent with previous studies, in both saliva fractions from OPC cases, we observed a large proportion with a mixed HPV-16 status. Surprisingly, there were two OPC cases that harboured only integrated HPV-16. Furthermore, the HPV-16 physical status in both saliva fractions and tumour biopsies of patients with OPC was positively correlated.

The major limitation of our study is the relatively small sample size of OPC cases. In addition, a qPCR based method is impotence to measure a single HPV integrated copy with multiple copies of episomal components when the Ct value of E2 and E6/7 was about similar. In summary, we have demonstrated the clinical and diagnostic utility of saliva as a noninvasive medium to investigate the HPV DNA prevalence, type distribution and physical status in OPC patients, regardless of salivary sampling methods. Further studies with larger sample sizes and additional robust methods for the salivary HPV-16 physical status examination will be needed to confirm the predictive value of this method. 


\section{Acknowledgements:}

We would like to thank Ms Dana Middleton and the staff at the ENT Department of the Princess Alexandra Hospital, Woolloongabba, Australia for their assistance in the recruitment of study patients and collection of clinical samples. We also thank Dr Gert Scheper (Janssen Vaccines and Prevention, Leiden, the Netherlands) and Dr Kurt Baeten (Janssen Diagnostics, Janssen Pharmaceutical, Beerse, Belgium) for technical and editorial assistance. CP is supported by QUT VC Fellowship. This work was supported by the Queensland Centre for Head and Neck funded by Atlantic Philanthropies, the Queensland Government. KDT salary is supported by the Janssen: Pharmaceutical Companies of Johnson \& Johnson.

\section{Conflict of Interest:}

The authors disclose no potential conflict of interest.

This article is protected by copyright. All rights reserved. 


\section{Figure legends:}

Figure 1: HPV-16 E2 (A) and E6/7 (B) relative copy number (Ct values) in salivary oral rinse and UWMS collected from OPC patients using qPCR. The fold change difference between E2 and E6/7 relative copy number in both saliva fractions (C) and tumour samples (D) collected from OPC patients.

Table 1: Patient demographics and characteristics

\begin{tabular}{|l|c|}
\hline \multicolumn{2}{|c|}{ Table 1: Participants demographic characteristics } \\
\hline Age (mean, range) & OPC patients (n 45$)$ \\
\hline Gender & $60.4,20-92$ \\
\hline Male & $42(93.3)$ \\
\hline Female & $3(6.7)$ \\
\hline Race and ethnicity & $43(95.6)$ \\
\hline Caucasian & $2(4.4)$ \\
\hline Other & \\
\hline Smoking status & $9(20.0)$ \\
\hline Smokers & $22(48.9)$ \\
\hline Ex-smoker & $14(31.1)$ \\
\hline Non-smoker & $0(0.0)$ \\
\hline Tumour characteristics & $0(0.0)$ \\
\hline AJCC TNM Stage I & $9(20.0)$ \\
\hline & $36(80.0)$ \\
\hline Stage II & \\
\hline & $26(57.8)$ \\
\hline Tumour anatomic sites & $10(22.2)$ \\
\hline Tonsil & $9(20.0)$ \\
\hline BOT & $34(75.6)$ \\
\hline Others & $10(22.2)$ \\
\hline p16INK4a status & $1(2.2)$ \\
\hline Positive & $3(6.7)$ \\
\hline Negative & \\
\hline N/A & \\
\hline Differentiation status & \\
\hline Well differentiated & \\
\hline
\end{tabular}

This article is protected by copyright. All rights reserved. 


\begin{tabular}{|l|c|} 
Well to moderately differentiated & $2(4.4)$ \\
\hline Moderately differentiated & $11(24.4)$ \\
\hline $\begin{array}{l}\text { Moderately to poorly } \\
\text { differentiated }\end{array}$ & $7(15.6)$ \\
\hline Poorly differentiated & $8(17.8)$ \\
\hline N/A & $14(31.1)$ \\
\hline
\end{tabular}

This article is protected by copyright. All rights reserved. 
Table 2: HPV type distribution in salivary oral rinse and UWMS collected from OPC patients using Hybribio 14 high risk-HPV real time PCR.

\begin{tabular}{|c|c|c|c|c|}
\hline \multirow[t]{2}{*}{$\begin{array}{l}\text { OPC } \\
\text { Sample }\end{array}$} & \multicolumn{2}{|c|}{ HPV-16 } & \multicolumn{2}{|c|}{$\begin{array}{c}12 \text { high risk HPV } \\
(31,33,35,39,45,51,52,56,58,59,66,68)\end{array}$} \\
\hline & $\begin{array}{l}\text { Salivary Oral } \\
\text { Rinse }\end{array}$ & $\begin{array}{c}\text { Unstimulated } \\
\text { whole mouth } \\
\text { saliva }\end{array}$ & $\begin{array}{l}\text { Salivary Oral } \\
\text { Rinse }\end{array}$ & $\begin{array}{c}\text { Unstimulated } \\
\text { whole mouth } \\
\text { saliva }\end{array}$ \\
\hline 1 & Yes & Yes & No & No \\
\hline 2 & Yes & Yes & No & No \\
\hline 3 & Yes & Yes & No & No \\
\hline 4 & Yes & Yes & No & No \\
\hline 5 & Yes & Yes & No & No \\
\hline 6 & Yes & Yes & Yes (Low) & Yes (Low) \\
\hline 7 & Yes & Yes & No & No \\
\hline 8 & Yes & Yes & Yes (Low) & Yes (Low) \\
\hline 9 & Yes & Yes & Yes & Yes \\
\hline 10 & Yes & Yes & No & No \\
\hline
\end{tabular}


Table 3: The physical status of HPV-16 in the salivary oral rinse, UWMS and tumour samples collected from OPC patients.

\begin{tabular}{|c|c|c|c|}
\hline & Episomal & Mixed & Integrated \\
\hline Salivary oral rinse samples $(\mathrm{n}=30)$ & $5(16.6)$ & $23(76.7)$ & $2(6.7)$ \\
\hline Unstimulated whole mouth saliva $(\mathrm{n}=30)$ & $1(3.3)$ & $27(90.0)$ & $2(6.7)$ \\
\hline Tumour samples $(\mathrm{n}=2)$ & $0(00)$ & $2(100)$ & $0(0)$ \\
\hline
\end{tabular}


Supplementary Table S1: Primer Sequences.

Supplementary Table S2: The detection of HPV-16 DNA in both saliva fractions collected from p16INK4a-negative and -positive OPC patients.

This article is protected by copyright. All rights reserved. 


\section{References:}

1. Ferlay J, Soerjomataram I, Dikshit R, et al. Cancer incidence and mortality worldwide: sources, methods and major patterns in GLOBOCAN 2012. Int J Cancer. 2015;136(5):E359386.

2. Blot WJ, McLaughlin JK, Winn DM, et al. Smoking and drinking in relation to oral and pharyngeal cancer. Cancer Res. 1988;48(11):3282-3287.

3. Gillison ML, Chaturvedi AK, Anderson WF, Fakhry C. Epidemiology of Human PapillomavirusPositive Head and Neck Squamous Cell Carcinoma. J Clin Oncol. 2015;33(29):3235-3242.

4. Sun CX, Bennett N, Tran P, et al. A Pilot Study into the Association between Oral Health Status and Human Papillomavirus-16 Infection. Diagnostics (Basel). 2017;7(1).

5. Gillison ML, Koch WM, Capone RB, et al. Evidence for a causal association between human papillomavirus and a subset of head and neck cancers. J Natl Cancer Inst. 2000;92(9):709720.

6. Michaud DS, Langevin SM, Eliot M, et al. High-risk HPV types and head and neck cancer. Int J Cancer. 2014;135(7):1653-1661.

7. Fakhry C, Zhang Q, Nguyen-Tan PF, et al. Human papillomavirus and overall survival after progression of oropharyngeal squamous cell carcinoma. J Clin Oncol. 2014;32(30):33653373.

8. Spector ME, Gallagher KK, Bellile E, et al. Patterns of nodal metastasis and prognosis in human papillomavirus-positive oropharyngeal squamous cell carcinoma. Head Neck. 2014;36(9):1233-1240.

9. Posner MR, Lorch JH, Goloubeva O, et al. Survival and human papillomavirus in oropharynx cancer in TAX 324: a subset analysis from an international phase III trial. Ann Oncol. 2011;22(5):1071-1077.

10. Chaturvedi AK, Engels EA, Pfeiffer RM, et al. Human papillomavirus and rising oropharyngeal cancer incidence in the United States. J Clin Oncol. 2011;29(32):4294-4301.

11. Klussmann JP, Weissenborn SJ, Wieland U, et al. Prevalence, distribution, and viral load of human papillomavirus 16 DNA in tonsillar carcinomas. Cancer. 2001;92(11):2875-2884.

12. Kim SH, Koo BS, Kang S, et al. HPV integration begins in the tonsillar crypt and leads to the alteration of p16, EGFR and c-myc during tumor formation. Int J Cancer. 2007;120(7):14181425.

13. zur Hausen H. Papillomaviruses in human cancers. Proc Assoc Am Physicians. 1999;111(6):581-587.

14. Ahn SM, Chan JY, Zhang Z, et al. Saliva and plasma quantitative polymerase chain reactionbased detection and surveillance of human papillomavirus-related head and neck cancer. JAMA Otolaryngol Head Neck Surg. 2014;140(9):846-854.

15. Qureishi A, Ali M, Fraser L, Shah KA, Moller H, Winter S. Saliva testing for human papilloma virus in oropharyngeal squamous cell carcinoma: A diagnostic accuracy study. Clin Otolaryngol. 2017.

16. Rogers NL, Cole SA, Lan HC, Crossa A, Demerath EW. New saliva DNA collection method compared to buccal cell collection techniques for epidemiological studies. Am J Hum Biol. 2007;19(3):319-326.

17. Steinau $M$, Reddy $D$, Sumbry $A$, et al. Oral sampling and human papillomavirus genotyping in HIV-infected patients. J Oral Pathol Med. 2012;41(4):288-291.

This article is protected by copyright. All rights reserved. 
18. Chai RC, Lim Y, Frazer IH, et al. A pilot study to compare the detection of HPV-16 biomarkers in salivary oral rinses with tumour p16(INK4a) expression in head and neck squamous cell carcinoma patients. BMC Cancer. 2016;16:178.

19. Yang $\mathrm{H}, \mathrm{Li} \mathrm{L}, \mathrm{Xie} \mathrm{LX}$, et al. Clinical validation of a novel real-time human papillomavirus assay for simultaneous detection of 14 high-risk HPV type and genotyping HPV type 16 and 18 in China. Arch Virol. 2016;161(2):449-454.

20. Canadas MP, Darwich L, Sirera G, et al. New molecular method for the detection of human papillomavirus type 16 integration. Clin Microbiol Infect. 2010;16(7):836-842.

21. Tang KD, Kenny L, Perry C, Frazer I, Punyadeera C. The overexpression of salivary cytokeratins as potential diagnostic biomarkers in head and neck squamous cell carcinomas. Oncotarget. 2017;8(42):72272-72280.

22. Pfaffe T, Cooper-White J, Beyerlein P, Kostner K, Punyadeera C. Diagnostic potential of saliva: current state and future applications. Clin Chem. 2011;57(5):675-687.

23. Ang KK, Harris J, Wheeler R, et al. Human papillomavirus and survival of patients with oropharyngeal cancer. N Engl J Med. 2010;363(1):24-35.

24. Wang $\mathrm{Y}$, Springer S, Mulvey $\mathrm{CL}$, et al. Detection of somatic mutations and HPV in the saliva and plasma of patients with head and neck squamous cell carcinomas. Sci Transl Med. 2015;7(293):293ra104.

25. Shin HJ, Joo J, Yoon JH, Yoo CW, Kim JY. Physical status of human papillomavirus integration in cervical cancer is associated with treatment outcome of the patients treated with radiotherapy. PLoS One. 2014;9(1):e78995.

26. Koskinen WJ, Chen RW, Leivo I, et al. Prevalence and physical status of human papillomavirus in squamous cell carcinomas of the head and neck. Int J Cancer. 2003;107(3):401-406.

27. Chen X, Gao L, Sturgis EM, et al. HPV16 DNA and integration in normal and malignant epithelium: implications for the etiology of laryngeal squamous cell carcinoma. Ann Oncol. 2017;28(5):1105-1110.

28. Deng Z, Hasegawa M, Kiyuna A, et al. Viral load, physical status, and E6/E7 mRNA expression of human papillomavirus in head and neck squamous cell carcinoma. Head Neck. 2013;35(6):800-808. 